\title{
An Approach for Designing and Implementing Evidence Management Systems
}

\author{
Dongping Gao ${ }^{1}$, Zhendong Niu ${ }^{2}$, Baosheng Zhang ${ }^{3}$, Nanning Zhang ${ }^{4}$ \\ ${ }^{1}$ Institute of Medical Information, Chinese Academy of Medical Sciences, Beijing, China \\ ${ }^{2}$ School of Computer Sciences, Beijing Institute of Technology, Beijing, China \\ ${ }^{3}$ Key laboratory of Evidence Science (China University of Political Science and Law), Ministry of Education, Beijing, \\ China \\ ${ }^{4}$ Hunan Tiandiren Law Firm, Hunan, China \\ E-mail: gaodp_gaodp@yahoo.com.cn
}

Received January 1, 2010; revised April 8, 2020; accepted August 7, 2010

\begin{abstract}
Generally speaking, the software of management systems in law field is based on the frames of events. However, we are going to study and develop the new software of management system in which the basic elements are evidence. This kind of software is often called evidence management system. Here we are supposed to present a designing plan and an implementing approach for the evidence management system in detail. Some functions such as global, dynamic and systematic managing of evidence can be implemented in this system. We attempt to provide a function of individual searching as well. Users may carry out multi-dimensional data analysis based on the information of our database of the management systems.
\end{abstract}

Keywords: Evidence, Evidence Management, Evidential View, Personal View, Statistical Analysis

\section{Introduction}

It is well known, fact-finding and law applications to facts are the two major tasks in the process of trial. Fact-finding is a process that uses evidence to prove the authenticity of a law case in a given case. Therefore, evidence is a cornerstone of achieving justice. In judicial practice, whether in criminal, civil or administrative cases, all activities depend on the fact investigations of cases. Effective management of evidence information can improve the efficiency and accuracy of fact-finding.

Now, in litigation in China, in the process of collections, classifications cross-examinations and certifications of evidence, empirical methods are basically used, and physical ways are used in the keep of evidence. As a whole, evidence management stays in paper files and hand-operating levels. In trials, evidence presentations, identifications, bearing witness, direct and cross-examinations, are the way by clerk recording. Judges can only depend on experience to judge and think in a broad array of evidence information. Whether judges admitting or excluding an item of evidence or making a final judicial decision lack the evidence that are the result after information processing. This not only wastes a lot of time, but also brings difficulties for judges in making comprehensive evaluations of evidence information.

\section{Related Researches}

So far, the results of theoretical level of evidence study are quite rich [1-3]. With the development of computer sciences, scholars have begun to dedicated to research information management systems, including expert systems of evidence [4], electronic evidence management systems [5,6], and artificial intelligence for judicial evidence as well [7]. There has been some information systems related to evidence management, such as "Comprehensive Evidence Management System” developed by Eden Company in Australia and "Forensic Science Evidence Management System" in UK. These management systems are designed primarily to meet the actual partial needs of the evidence management, but are not comprehensive, dynamic, systematic studies.

In China, researches for evidence management systems have not yet started, related studies include the following:

1) The initial work in china for legal expert systems can go back to eighties of the 20th century by Qian XueSen. Professor Qian published a series of articles 
$[8,9]$, they can treated as a fundamental stone for the development of our legal expert systems.

2) Hu Zhao, Zhou Zongyi, Wang Hongjie from Schools of Law and computer science in Sun YAT-SEN University, who co-developed the "Lawyers' Office Automation System” in 1993. The system has functions of file management and legal information management [10].

3) TingGuang Zhao presided over the development of “A Practical Expert System in Criminal Law". The system is divided into three subsystems, saying that "Consultation Retrieval System”, “Assisted Qualitative System”, “Auxiliary Sentencing System” [11].

\section{Our Evidence Management System (EMS)}

\subsection{A Design for the Evidence Management System}

In order to achieve a comprehensive, dynamic, systematic management of evidence and to realize a multi-dimensional statistical analysis on databases, the evidence management system in this paper is designed to have the following modules: registration module, the case information input module, query module, statistics module and output judgments module. Details are shown in Figure 1.

Registration module checks the users who are going to log on the system for whether they are pre-registered individuals. The ways of authentication include client applications and manual authentication certificate.

The case information input module includes two input sub-modules for the basic and the detailed information of cases respectively. The input sub-module for the basic information of cases records case names, case numbers, courts which accepted to deal with the respective cases and so on. The input sub-module for the detailed information of cases accomplishes two functions of the personnel information input and the evidence information input. It consists of the following five parts: 'the basic

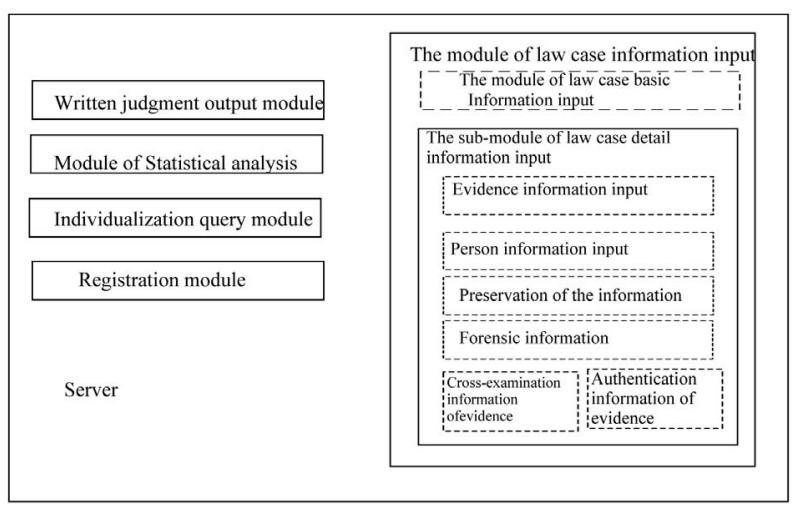

Figure 1. Design of modules of the evidence management system information of evidence' module-to input basic information about evidence; 'preservation of the information' module-to input the information preservation of evidence; forensic information module-to input the proof information of evidence; cross-examination module-to input the cross-examination information of evidence; authentication module-to input the authentication information of evidence.

The query module provides systematic individualized inquiry service for users by matching between a wildcard and the content inside the databases.

The statistic module enables statistical analysis according to the information in databases. Statistical rules are flexible. Users can choose different fields for the statistical analysis.

The judgment output module is automatically associated with the type of cases in which users selected the property (criminal, civil or administrative) of each case in the basic information input module. Different cases use different templates and then output the respective judgments automatically.

\subsection{The Module of Law Case Information Input}

The law case detail information input sub-module is one of the core modules in the system. The functions of this module are achieved by Struts2, Hibernate, Ajax technologies. This module includes filling in personal information and evidence information through a person table, to record all personnel and staff information through a type table. Personnel information implement functions of add, delete, modify, and other operations through an action called AccuserList. Persons may provide appropriate evidence, and the evidence is associated with the 'person table'. On the session, users can add, delete, and revise evidence and other operations. The entire pages use Struts2 tag, such as time control. At the same time, we use Ajax technology to achieve cascading drop-down menu 5. All filling information is designed according to the process of the trial.

Adding processes of each evidence includes almost all of the relevant content of the "People's uniform rules of evidence" [12]. In addition to itself information of evidence, such as evidence types, evidence specifications and so on, the input process of evidence information can be shown in the Figure 2.

Forensic information sub-module includes: obtained evidence in accordance with duties and in accordance with applications.

Cross-examination sub-module includes: the content of cross-examination, the reasons of cross-examination, discerning the false from the genuine (truth) and etc.

Authentication sub-module includes: whether the evidence and facts of a case are relevant and admissible; 
whether the source of evidence is legitimate and reliable; whether the content of evidence is true; the admission of evidence whether meet the requirements of fair trial, whether injury or injustice of the verdict impacting on parties is substantially exceed its probative value; whether it is evidence which cannot been admitted alone.

\subsection{Cross-Transfer between Person View and Evidence View}

In Evidence management system, we take into account person interrelated with evidence, the case detail information input sub-module is designed into two display modes. In one display mode, personal information is input clues; in the other, evidence information is input clues. The two display modes can switch and they can realize the same functionality. In evidence management system, we use Ajax technology to achieve person and evidence displays of the dynamic switching. Users can choose display modes by individual preferences.

In the personal clue display input mode, the input clues is personal information, a person is with information on their own information and evidence information. Evidence information for the persons referred to him who provided evidence. Other words, in this input mode, an item of evidence information is attribute information as person provided. In evidence display input mode, the evidence is the clew of the input process, and provider is one property of evidence, that is personnel information.

\subsection{Individualization Query Module}

Query module provides a convenient functional of inquiries for users. Submitting data and returning query results in query module is achieved by Ajax technology without refreshing the page. Query module provides three query services:

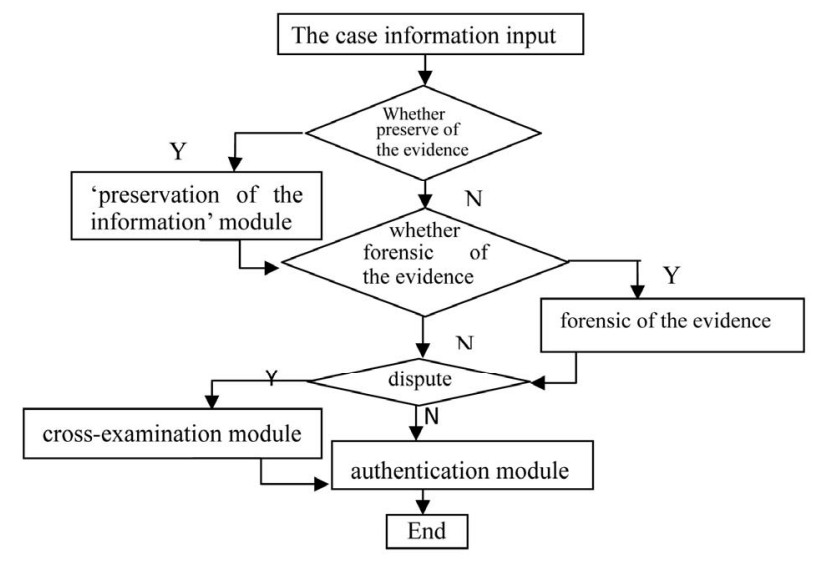

Figure 2. Add the evidence information.
1) Quick query: the query condition is time, such as the last week input cases, the last month input cases, and even the last year cases input, and so on. If a user clicks on each specific query requests, it can show the basic information list of cases. If you click on the corresponding list of basic information fields you can further view the case details.

2) Key words search: users can input their knowledge of a case as a keyword query, such as plaintiff's names, case names, case processing time and so on. Displayed pages are the case list with those key words. If you click on the list of information, you can further view the case details.

3) Advanced search: this allows users to process multi-keyword queries and Boolean operations on the key words to search.

\subsection{Module of Statistical Analysis}

The main function of the statistics module is based on information from the database to achieve statistical analysis. Technically, the statistics module establishes charts by JFreeChart, and creates charts of data sources by accessing to the database, the specific steps are as following:

1) Connect to the database;

2) Obtain corresponding data by dynamic cross tables technology;

3) The establishment of statistical graphic data set (DataSet);

4) Create table (JFreeChart) object using data set object;

5) Using JFreeChart correlation method to response graphic display.

In EMS, statistical module uses columns, line graphs and pie charts, which are typically three statistical result display methods. Specifically, the system statistics module consists of statistical analysis of whole cases, case precise statistical analysis, case statistical analysis by timing, personnel comprehensive statistical analysis, and comprehensive statistical analysis of the evidence.

Statistical analysis of the whole cases achieves the statistical analysis of overall time (period), place (the court) and the number of cases. Generally the results are usually displayed with a hot pie chart, and the type and quantity of information can been further demonstrated in this way.

The service of accurate statistical analysis of cases achieves statistical analysis of a variety of different combinations with types of cases, time, places (the court) and the number of cases. Generally, the statistical results are showed in the form of columnar diagram (see Figure 3). 


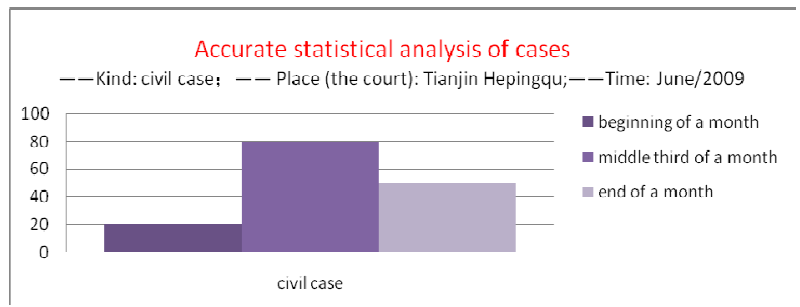

Figure 3. Accurate statistical analysis of cases.

Temporal statistical analysis of cases achieves statistical analysis of a variety of different combinations with types of cases, different time (period), places (the court) and the number of cases. Generally statistical results are displayed by the form of line chart (see Figure 4).

The statistical methods of person achieves statistical analysis of a variety of different combinations with case types, time (period), places (the court), ages, kinds of education, genders, number of cases of various personnel types. Generally, the statistical results are displayed by column graphs. The comprehensive statistical analysis of evidence achieves statistical analysis that a variety of different combinations with different time (period), different types of evidence, whether the evidence has been preserved and collected, and the number of cases.

Users can develop statistical criteria according to their own statistical requirements, for example, they can gain statistic results on the ratio of the various types of cases in a certain period, or in a certain area.

\subsection{Written Judgment Output Module}

After input all information of a case, the system can create automatically a written judgment. It makes judgments and case information directly linked. This can greatly reduce the workload of the staff and makes written judgments be connected with the information of cases. Judgment output module is achieved by Simple XML serialization framework, using fully configured XML structures. Objects of the case are serialized into XML data streams. Through XSL, and application of a number of predefined templates, the XML of case objects can be transferred into HTML documents, and it is actually converted into case word documents, in order to achieve the output of the court cases.

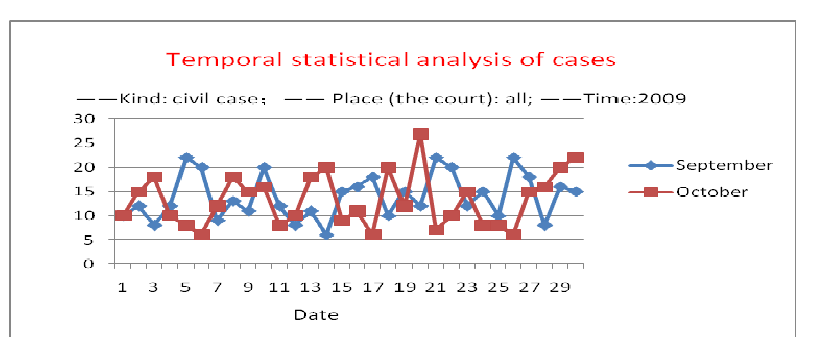

Figure 4. Temporal statistical analysis of cases.

\section{Conclusions}

We designed an evidence management system that can achieve fully dynamic and systematic management for evidence by segmenting the content of cases into a level of evidence. Person display and evidence display are interrelated, a person is accompanied with "evidence" field, the evidence is accompanied with a "provider" field, and the person display and the evidence display can transform each other. The evidence management reflects and integrates all the provisions of the proposed draft and argument of the "People's uniform rules of evidence". The system can be used for the actual work in our judicial system. The statistical analysis module in the management system enables comprehensive statistical analysis of a variety of fields. It can help for theoretical study and judicial practice.

The system may also provide a platform of comprehensive analysis for the theoretical study of evidence. It may have a certain degree of theoretical significance for the development of evidence science and management theory. It seems possible to have practical values as well for decision-making to improve the state of social information management system.

\section{Acknowledgements}

This article is sponsored by the National Natural Science Foundation project "evidence of the fact-finding mode Management” (project approval number: 70873134) and evidence Ministry of Education Key Laboratory Open Foundation of 2008 (project approval number: 08KFKT004) funding.

\section{References}

[1] M. H. Shao, J. J. Hwang and S. S. Wu, “A Transactional-Cycle Approach to Evidence Management for Dispute Resolution,” Information \& Management, Vol. 42, No. 4, 2005, pp. 607-618.

[2] Shepherd and Jonathan, "The Production and Management of Evidence for Public Service Reform, Evidence \& Policy," A Journal of Research, Debate and Practice, Vol. 3, No. 2, May 2007, pp. 231-251.

[3] M. MacCrimmon and P. Tillers, "The Dynamics of Judicial Proof: Computation, Logic, and Common Sense,” Physica-Verlag, Heidelberg, 2002.

[4] W. Schwarzer and J. Cecil, "Management of Expert Evidence," In Reference Manual on Scientific Evidence, 2000.

[5] J. Pfeffer and R. I. Sutton, "Evidence-Based Management,” Harvard Business Review, Vol. 1, 2006.

[6] D. Lee, "Electronic Evidence Management: From Creation to Litigation,” Fios Publisher, 2005.

[7] P. Tillers, "Introduction: A Personal Perspective on Arti- 
ficial Intelligence and Judicial Proof,” Cardozo Law Review, Vol. 22, 2001.

[8] X. S. Qian, "The Task and Methods of Law Systems Engineering," Science and Technology Management Research, Vol. 4, 1981.

[9] X. S. Qian, "Modern Science and Technology and Law and the Legal System,” Political Forum, Vol. 3, 1985.
[10] J. G. Yang and M. F. Luo, "Legal System Engineering,” Sun Yat-Sen University Press, China, 1996, pp. 344-349.

[11] T. G. Zhao, "User Manual of Practical Criminal Law Expert System,” New Concept Software, 1993.

[12] B. S. Zhang, "Uniform Provisions of Evidence of the People's Court," Institute of Evidence Law and Forensic Science, China. 